\title{
Multiscale modelling of gas and moisture transport during refrigerated storage of fruit
}

\author{
$\underline{\text { B. M. Nicolai }}^{1}$, N.T. Anh ${ }^{1}$, Q.T. Ho ${ }^{1}$, H.K. Mebatsion ${ }^{1}$, F. Mendoza ${ }^{1}$, P. Verboven ${ }^{1}$, B.
} Verlinden $^{1}$ and $\mathrm{M}$. Wevers ${ }^{2}$

${ }^{1}$ Flanders Centre of Postharvest Technology / BIOSYST - MeBioS, Catholic University of Leuven, de Croylaan 42, B-3001 Leuven, Belgium; bart.nicolai@biw.kuleuven.be

${ }^{2}$ Department of Metallurgy and Materials Engineering (MTM), Catholic University of Leuven, Kasteelpark Arenberg 44, B-3001 Leuven, Belgium

\begin{abstract}
The success of controlled atmosphere storage of fruit critically depends on the gas composition of the storage atmosphere. Gas transport models can be used advantageously to evaluate the effect of gas composition on product quality and to optimise and control the storage process. In this paper we will discuss gas and moisture transport models of increasing complexity. We will focus on recent advances in multiscale transport models. Such models are basically a hierarchy of models which describe the transport phenomena at different spatial scales in such a way that the submodels are interconnected. We will pay particular attention to methods to measure product topology and material properties at the micro- and nanoscale.
\end{abstract}

Keywords : distributed models, transport properties, gas, fruit, controlled atmosphere, storage, multiscale

\section{Introduction}

Apples and pears are perishable commodities whose storage life depends on, amongst other factors, the respiratory activity of the fruit. Commercial storage is essentially based on reducing the respiration as much as possible without causing adverse effects such as offflavours and storage disorders due to fermentation processes. In controlled atmosphere storage, the fruit are hereto stored at a low temperature (typically around $0^{\circ} \mathrm{C}$ ) in combination with a reduced $\mathrm{O}_{2}$ and increased $\mathrm{CO}_{2}$ partial pressure (so-called "Controlled Atmosphere (CA) storage"). In this way the storage life of some apple and pear cultivars can be extended to up to one year.

For a proper operation of the controlled atmosphere cool rooms a more profound insight in the metabolic reactions and transport processes inside the stored fruit is mandatory. A mechanistic modelling approach seems most appropriate, as it provides more insight into the physiological phenomena related to storage disorders. In this paper we will present an overview of recent advances in modelling of gas and moisture transport processes in fruit, with particular attention to multiscale models.

\section{Controlled Atmosphere storage}

In controlled atmosphere storage the $\mathrm{O}_{2}$ and $\mathrm{CO}_{2}$ concentration are maintained at a welldefined set point level. This set point is often close to the anaerobic compensation point, the $\mathrm{O}_{2}$ partial pressure at which the $\mathrm{CO}_{2}$ production rate is at its minimum. The optimal gas composition is critical, as too low an $\mathrm{O}_{2}$ partial pressure in combination with too high a $\mathrm{CO}_{2}$ 
partial pressure induces a fermentative metabolism in the fruit (Beaudry, 1999; Yearsley et al., 1996). This causes off-flavours (e.g., ethanol) and storage disorders (e.g., core breakdown in pear). For this reason, the $\mathrm{O}_{2}$ and $\mathrm{CO}_{2}$ partial pressure in commercial cool stores is kept at a safe and steady value. The optimal storage temperature depends on the produce and is in general the lowest temperature preventing the produce from chilling injury or freezing damage.

The $\mathrm{O}_{2}$ can be reduced by respiration as such, and by injecting $\mathrm{N}_{2}$ gas produced from ordinary air by removing ("scrubbing") the $\mathrm{O}_{2}$ and $\mathrm{CO}_{2}$. Storage under controlled atmosphere is widely used to store all kinds of horticultural produce. In ultra low oxygen (ULO) the $\mathrm{O}_{2}$ concentration is below $2 \%$. Some optimal gas compositions for long term storage of fruit and vegetables are summarised in Table 1.

Table 1. Optimal gas compositions for long term storage of apple and pear cultivars (Kader and Seaver, 2001)

\begin{tabular}{llll}
\hline Cultivar & $T\left({ }^{\circ} \mathrm{C}\right)$ & $\mathrm{O}_{2}(\%)$ & $\mathrm{CO}_{2}(\%)$ \\
\hline Apple & & & \\
Braeburn & 0.5 & 3 & $<1$ \\
Cox's Orange Pippin & 4 & 1.3 & 0.7 \\
Golden Delicious & 0.5 & 2.5 & 1 \\
Red Delicious & 0 to 0.5 & 2.5 & 4.5 \\
Jonagold & 1 & 1 & 4.5 \\
Pear & & & \\
Conference & -1 & 2.5 & 0.7 \\
Packham's Triumph & -0.5 & 1.5 & 2.5 \\
Williams Bon Chretien & 0 to -0.5 & 1 & 0 \\
\hline
\end{tabular}

Modified atmosphere packaging is a technique in which the produce is kept fresh for a longer period, using a gas permeable foil allowing, to some extent, gas exchange between the internal micro atmosphere in the package and the external atmosphere. The micro atmosphere is usually generated through respiration of the produce, but often a gas mixture is injected into the package to obtain a faster equilibrium.

\section{Gas transport}

If the local gas composition inside the fruit is too extreme, storage disorders may occur. An example of such disorders is core breakdown. This storage disorder is characterised by the development of brown tissue which will further develop into cavities so that the fruit can no longer be commercialised. The harvest time, the $\mathrm{O}_{2}$ and $\mathrm{CO}_{2}$ concentration in the cool room, the storage temperature, the size of the pear and their interactions are the most critical factors which affect the development of the disorder. The antioxidant content also affects the susceptibility of the pear to core breakdown. The exact mechanism of the disorder is not well understood, but it is believed to be caused by an imbalance in the energy metabolism in the 
cells due to too low an $\mathrm{O}_{2}$ or too high a $\mathrm{CO}_{2}$ concentration. This leads to membrane damage and decompartmentalisation. Phenolic compounds are then enzymatically oxidised to brown coloured compounds. Eventually, water diffuses from the centre towards the boundary of the pear and a cavity is created.

As limited gas transport inside the fruit is probably the prime cause of disorders such as core breakdown, several attempts have been made to model this process. Models to describe gas exchange of fruit and vegetables are usually based on enzyme kinetics according to Michaëlis-Menten:

$$
\frac{d}{d t}\left[O_{2}\right]=-\frac{m}{V} V_{O_{2}, \max } \frac{\left[O_{2}\right]}{K_{m}+\left[O_{2}\right]}
$$

where $m$ is the mass of the fruit, $V$ the volume of the enclosure in which the fruit is kept, $V_{O_{2} \text {,max }}$ the maximum oxygen consumption rate, and $K_{m}$ the Michaëlis-Menten constant or the concentration at which its activity is half of $V_{O_{2}, \max }$.

Equation (1) disregards transport phenomena in or at the surface of the fruit. However, the critical partial pressures beyond which undesireable phenomena develop are often much higher then one would expect from biochemistry. For example, cytochrome oxidase has a $K_{m}$ which is orders of magnitude below the typical $K_{m}$ of intact fruit $(1-3 \mathrm{kPa})$.

This suggests an oxygen gradient inside the fruit in such a way that the local oxygen partial pressure in the center of the fruit is much lower than in the storage atmosphere. This observation inspired Mannapperuma et al. (1991) and later on Lammertyn et al. (2003a) to develop a gas exchange model based on chemical reaction kinetics. The model is essentially a system of two coupled diffusion equations, one for $\mathrm{O}_{2}$ and one for $\mathrm{CO}_{2}$ which are coupled through the respiration kinetics

$$
\begin{aligned}
& \frac{\partial}{\partial t}\left[\mathrm{O}_{2}\right]=D_{\mathrm{O}_{2}} \nabla^{2}\left[\mathrm{O}_{2}\right]-V_{\mathrm{O}_{2}} \\
& \frac{\partial}{\partial t}\left[\mathrm{CO}_{2}\right]=D_{\mathrm{CO}_{2}} \nabla^{2}\left[\mathrm{CO}_{2}\right]+R Q_{\text {cell }} V_{\mathrm{O}_{2}} \\
&+\frac{V_{m, f, C O_{2}, \text { cell }}}{\left(1+\frac{\left[\mathrm{O}_{2}\right]}{K_{m, f, Q_{2}, \text { cell }}}\right)} \\
& V_{\mathrm{O}_{2}}=\frac{V_{m, Q_{2}, \text { cell }}\left[\mathrm{O}_{2}\right]}{\left(K_{m, Q_{2}, \text { cell }}+\left[\mathrm{O}_{2}\right]\right)\left(1+\frac{\left[\mathrm{CO}_{2}\right]}{K_{m n, \mathrm{CO}, \text {,eell }}}\right)}
\end{aligned}
$$

with $D_{O_{2}}$, the gas diffusivity of $\mathrm{O}_{2}$ in pear tissue; $V_{O_{2}}$, the $\mathrm{O}_{2}$ consumption rate, $\nabla^{2}$, the Laplace operator; $V_{m, Q_{2}, \text { cell }}$, the maximum cellular aerobic $\mathrm{O}_{2}$ consumption rate; $K_{m, O_{2}, \text { cell }}$, the 
tissue Michaelis-Menten constant and $V_{m n, \mathrm{CO}_{2}, \text { cell }}$, the tissue Michaelis-Menten constant for non-competitive inhibition of $\mathrm{CO}_{2}$ on $\mathrm{O}_{2}$ consumption; $D_{\mathrm{CO}_{2}}$, the $\mathrm{CO}_{2}$ diffusivity in pear tissue; $V_{\mathrm{CO}_{2}}$, the $\mathrm{CO}_{2}$ production, $R Q_{\text {cell }}$, the tissue respiration quotient; $V_{m, f, \mathrm{CO}_{2}, \text { cell }}$, the maximal tissue fermentative $\mathrm{CO}_{2}$ production rate; and $K_{m, f, O_{2} \text {, cell }}$, the inhibition constant of $\mathrm{O}_{2}$ on the tissue fermentative $\mathrm{CO}_{2}$ production. Appropriate boundary conditions need to be supplied as well (Lammertyn et al., 2003a and 2003b).

The couple (2)-(3) of nonlinear partial differential equations cannot be solved analytically, and numerical solution is required. Hereto the equations are discretised over a mesh of discretisation points - so-called nodes - to yield a large system of ODE couples (one equation for $\mathrm{O}_{2}$ and one for $\mathrm{CO}_{2}$ ) which describe the evolution of $\mathrm{O}_{2}$ and $\mathrm{CO}_{2}$ in these nodes. The ODE couple in a particular node is also coupled with that in the neighbouring nodes, and, as a consequence, all ODEs must be integrated in the time domain simultaneously. Several discretisation methods exist, including the finite difference, finite element and finite volume method, and the reader is referred to the literature for details about them (e.g., Nicolaï et al., 2001). It is important to note that commercial software (e.g., Femlab, Comsol AB, Götebörg, Sweden) exists which simplifies the implementation of numerical discretisation considerably and brings it into reach of a postharvest engineer who only needs to have some affinity with engineering mathematics.

Based on this model Lammertyn et al. (2003a, 2003b) computed three-dimensional $\mathrm{O}_{2}$ and $\mathrm{CO}_{2}$ profiles in Conference pears for various storage conditions. They observed that the predicted contours of the simulated $\mathrm{CO}_{2}$ and $\mathrm{O}_{2}$ profiles corresponded very well with the contours of core breakdown - a CA related disorder characterised by brown areas and eventually tissue breakdown and cavities - as observed by means of Magnetic Resonance Imaging (MRI) and X-ray Computer Tomography (CT) scans of the pears. The authors also carried out a simulation study to evaluate the effect of cooling management. They were able to show that delaying the application of controlled atmosphere conditions until three weeks after the beginning of the cooling period was beneficial to reduce the incidence of core breakdown (Lammertyn et al., 2003b). Ho and al. (2006) extended the model of Lammertyn et al. (2003a and 2003b) with a permeation term and an equation for $\mathrm{N}_{2}$ transport. A very good correspondence between measured and predicted gas partial pressures was obtained. Typical contours of $\mathrm{O}_{2}$ and $\mathrm{CO}_{2}$ are shown in Figure 2.

Many problems remain, though. Diffusion coefficients have been shown to vary considerably inside the fruit tissue (Schotsmans et al., 2003; Ho et al., 2005). This variation is certainly linked to the histology of the tis sue, but this relationship is not well understood. Further, it would be beneficial to be able to evaluate the effect of the local gas conditions on metabolic fluxes through metabolic network models. Such models include much more details about the actual biochemistry than the currently used Michaëlis-Menten models and would be useful to understand the biochemical causes of disorders such as core breakdown. This disorder is associated with membrane degradation processes which are believed to be related to impaired metabolic fluxes (Veltman et al., 1999). Finally, the model of Lammertyn et al. (2003a) essentially describes the metabolic gas distribution in the intercellular space - the actual gas concentration inside the cells is most probably lower. This is indicated by the $\mathrm{Km}$ value for respiration which was determined by the authors and which was still larger than typical $K_{m}$ values for cells and certainly cytochrome oxidase. Multiscale models might be appropriate to study these effects. 
(a)

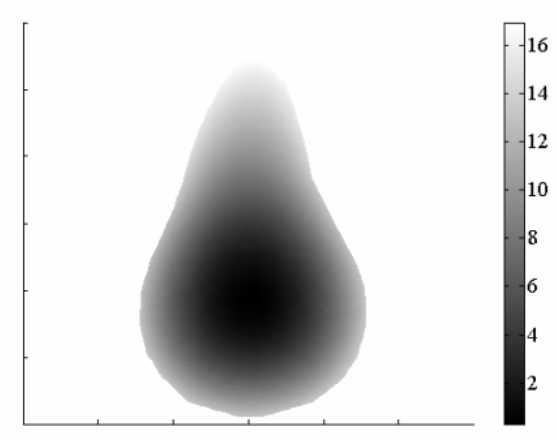

(b)

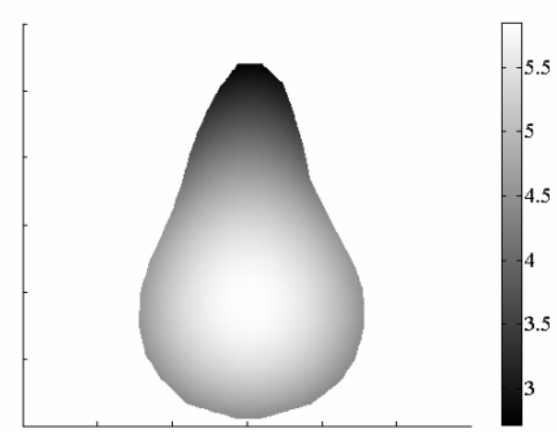

Fig. 2. Contour plots of $\mathrm{O}_{2}$ (a) and $\mathrm{CO}_{2}$ (b) partial pressure in Conference pear stored at $20^{\circ} \mathrm{C}$, $20 \mathrm{kPa} \mathrm{O}_{2}, 0 \mathrm{kPa} \mathrm{CO}$ and $80 \mathrm{kPa} \mathrm{N}_{2}$.

\section{Moisture transport}

Fruit always looses water during storage. This results in economic (mass) and quality (shrivel) losses. The cuticle which covers the fruit epiderms acts as a natural water impermeable barrier. Epidermal cells are similar to parenchyma cells but are smaller, have a larger aspect ratio and more tightly packed. The bulk of the fruit consists of parenchyma cells which are essentially semipermeable phospholipid membranes filled with cytoplasm and surrounded by a primary cell wall which is typically about $3 \mu \mathrm{m}$ thick and which consists of cellulose fibrils embedded in a pectin matrix. In between the cells there is a pectin layer called middle lamella which glues the cells together. The most important plant cell organel is the vacuole which is surrounded by a phospholipid membrane and which typically covers almost the entire cell volume. The cytoplasm of neighbouring cells are connected through plasmodesmata - narrow channels that act as intercellular cytoplasmic bridges to facilitate communication and transport of nutrients between plant cells. The plasmatic membrane and also the membranes of all intracellular organels contain proteins - the so-called aquaporins - which serve as additional passive water transport channels. Vascular bundels connect the stem with the calyx. They consist of xylem, responsible for water translocation in the plant, and phloem, responsible for the movement of most of the organic compounds. The conducting cells of the phloem contain cytoplasm and are joined end-to-end, thus forming a low-resistant transport system through the plant. The conducting cells (or vessel members) of the xylem, however, have lost their protoplast and the remaining cell walls form a long vessel. The end walls of individual vessel members are perforated to facilitate water transport under a hydrostatic pressure gradient. The xylem also contains xylem fibers that are long thin dead cells with a strongly lignified cell wall. Because of these transport structures, the cytoplasm of the apple cells form a interconnected spatial region which is called the symplast. The cell walls and the 
intercellular space form another region through which water can be transported and which is called the apoplast (Nobel, 1991).

Moisture gradients in fruit (apple, pear) have been modelled by means of Fick's second law of diffusion and subsequently validated by means of MRI (Nguyen et al., 2005). However, fruit shrink when they loose moisture, and the coupled hygromechanical problem is notably difficult to solve. Further, moisture in fruit is not free but entrapped in cells and its behaviour is determined by its thermodynamic state. The latter is affected by osmotic, turgor and capillary effects.

\section{Multiscale modelling}

From the preceeding sections it is clear that, unlike traditional engineering materials, fruit tissue has a complex fine structure. Nature has designed cellular tissue in such a way as to facilitate transport of metabolic gases and water. As a consequence, the continuum hypothesis does not hold anymore and a new modelling paradigm is required to describe the mechanics of deformation of vegetative tissue. The availability of such models would lead to increased insight into gas and moisture transport processes in fruit during storage.

A multiscale model consists of a hierarchy of models, which describe the mechanical behaviour at different spatial scales in such a way that the submodels are interconnected. It is convenient to distinguish three length scales:

- The macroscale addresses the fruit as such $\left(10^{-3}-10^{-2} \mathrm{~m}\right)$. At this scale the fruit is considered as a continuum which consists of different materials - skin and flesh.

- At the mesoscale, the actual topology of the fruit tissue incorporating the topology of the intercellular space and cell arrangement is considered $\left(10^{-5}-10^{-3} \mathrm{~m}\right)$. This involves the different types of tissue inside the fruit but also microscopic features such a lenticells and cracks in the wax layer which may affect gas and moisture transport and mechanical behaviour.

- The microscale is the cellular scale $\left(10^{-6}-10^{-4} \mathrm{~m}\right)$. Cell features such as the cell wall, the middle lamella and the vacuole are important for both moisture transport as well as mechanical behaviour.

In the multiscale modelling paradigm, numerical experiments are carried out at the smallest scale under consideration to compute apparent parameters, which are then used in a model which operates at a coarser scale. This process is repeated until the largest scale - typically a continuum-type model - is reached. At this scale, the macroscopic response variables of interest - fruit deformation under loading, water loss - are computed using apparent properties derived from the smaller scale model by a mathematical procedure called homogenisation (Panasenko, 2005; Hassani and Hinton, 1999). The reverse downscaling procedure is called localisation and can be used to investigate how the calculated macroscopic response variables affect microscopic events such as failure of cells. The concept of multiscale modelling is entirely new in postharvest technology and even plant physiology.

The development of such models is, however, complicated because of several reasons. First, the characterisation of the structure at the cellular and subcellular scale is not trivial (Schneider et al. 2002). X-ray computer tomography (CT) has been used to visualise and model the internal pore space of a variety of vegetative materials with a resolution limited to about 10 micrometers (Kuroki et al., 2004). As an example, in Fig. 3. the 3D pore space of apple parenchyma tissue is visualised by means of X-ray microfocus CT. Such models can readily be meshed for finite element analysis of gas or moisture transport. 
Measurements of mechanical and transport properties at the microscale level are difficult to perform, and few experimental techniques are available. Turgor pressure measurements can be carried out on individual cells in a tissue using the pressure probe (Hüsken et al., 1978). However, application of the technique to higher plant tissue consisting of small cells requires high technical skills. Nano-indentation techniques seem promising to determine mechanical properties on cellular scale, but have not been explored on individual fruit cells.

Model validation at meso and microscale is challenging. Experiments with well-defined tissues with known internal turgor pressure have to be carried out to validate the predicted mechanical behaviour. Measurement on tissue level require a sufficiently detailed, three dimensional understanding of tissue structure and deformations, and hence advanced imaging techniques suitable for the testing of biological material in its native hydrated state (Hepworth and Bruce, 2000; Donald et al., 2003, Zdunek et al., 2004).

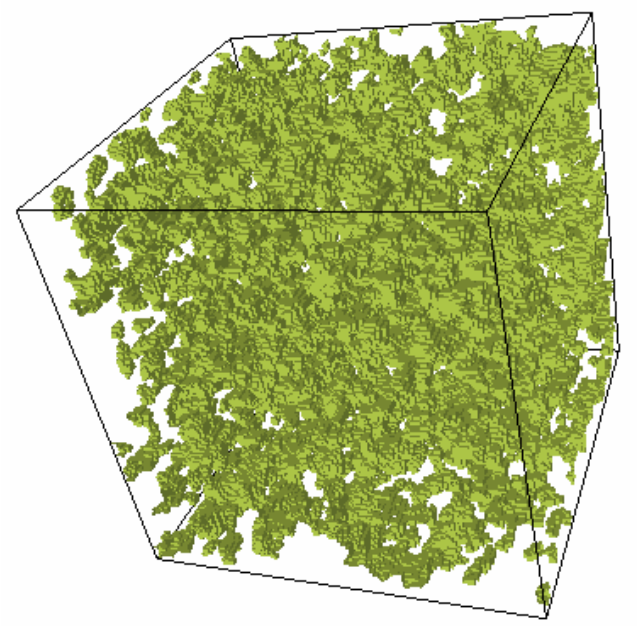

Fig. 3. 3D pore-cellular material surface of apple parenchyma tissue (Victor Mendoza, unpublished) obtained by means of X-ray microfocus CT

Multiscale models were first introduced in postharvest engineering by Veraverbeke et al. (2003a and b) who used a multiscale approach to model moisture loss of apple. They measured first moisture transport properties of the different materials (cutin, wax, parenchyma tissue) and incorporated them subsequently into a mesoscale continuum model which took into account the different components of the cuticle such as cutin and wax, and epidermal structures such as cracks and lenticels. The mesoscale model was used to estimate apparent diffusion properties of the skin which were then introduced in a macroscopic continuum model. This model was used successfully to understand the differences in moisture loss between Jonagold and Elstar apples which were shown to be related to the presence of cracks in the wax layer of Elstar which did not disappear during storage as was the case with Jonagold.

Current research focusses on gas transport in fruit tissue. As an example, in Fig. 4. the $\mathrm{O}_{2}$ concentration in apple parenchyma tissue during a numerical experiment is shown. It is clear that the oxygen concentration in the intercellular space is considerably larger than within the cells. Such information can be used to estimate apparent transport properties which can be inserted in a macroscale continuum model. 


\section{Conclusions}

Optimisation of controlled atmosphere storage requires a profound insight in the gas and moisture transport properties in the fruit during storage. While continuum type transport models in general describe the transport processes well, they involve apparent material properties which need to be obtained experimentally. Also, they do not lead to increased insight. Multiscale modelling addresses the transport processes at different spatial scales and integrates the models which each operate at a specific scale. While such an approach clearly is advantageous, many problems still have to be solved such as obtaining the tissue topology at smaller scales and measuring the model parameters experimentally.

(a)

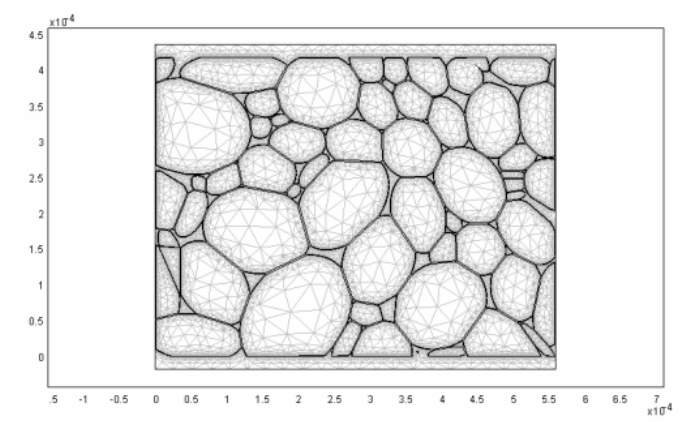

(b)

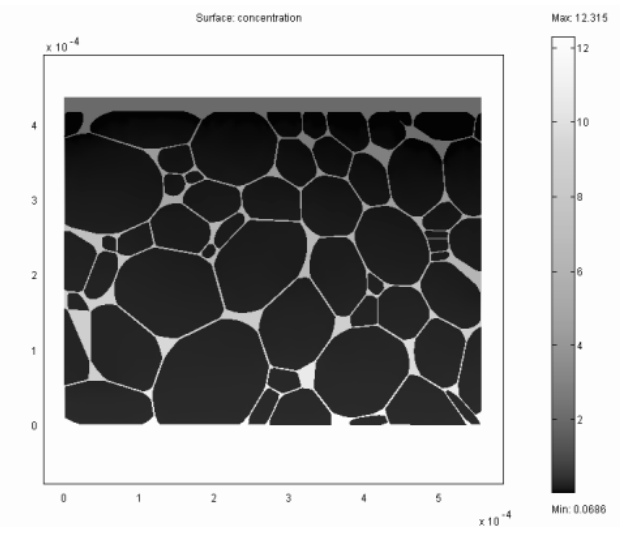

Fig. 4. Finite element mesh (a) and simulation (b) of the $\mathrm{O}_{2}$ concentration in pear parenchyma tissue (Quang Tri Ho, unpublished); concentrations in $\mathrm{mol} / \mathrm{m} 3$.

\section{Acknowledgements}

The authors would like to acknowledge the Flanders Fund for Scientific Research (FWOVlaanderen) (project G.0200.02) and the K.U.Leuven (projects IDO/00/008, OT 04/31, scholarships of author T.Q. Ho and N.T. Anh) for financial support. Author Pieter Verboven is Postdoctoral Fellow with the Flanders Fund for Scientific Research (FWO-Vlaanderen).

\section{References}

Beaudry R.M. (1999). Effect of $\mathrm{O}_{2}$ and $\mathrm{CO}_{2}$ partial pressure on selected phenomena affecting fruit and vegetable quality. Postharvest Biology and Technology. 15, 293-303.

Burton W.G. (1982). Post-harvest physiology of food crops. Longman, London and New York. 
Donald, A.M., F.S. Baker, A.C. Smith, K.W. Waldron (2003). Fracture of plant tissues and walls as visualized by environmental scanning electron microscopy. Annals of Botany, 92, 73-77.

Hassani B. and E. Hinton (1999). Homogenization and Structural Topology Optimization: Theory, Practice, and Software. Springer Verlag, New York.

Hepworth, D.G., D.M. Bruce (2000). Measuring the deformation of cells within a piece of compressed potato tuber tissue. Annals of Botany. 86, 287-292.

Ho, Q.T., B.E. Verlinden, P. Verboven, S. Vandewalle, B.M. Nicolaï (2006). Gas permeation in pear fruit. Submitted.

Ho, Q.T., B.M. Verlinden, P. Verboven, and B.M. Nicolaï (2005). Measurement of space dependent gas diffusion properties of pear tissue using optical sensors. Submitted.

Hüsken D., E. Steudle, U. Zimmerman (1978). Pressure probe technique for measuring water relations of cells in higher plants. Plant Physiology. 61, 158-163.

Kader, A., D. Seaver (2001). Optimal Controlled Atmospheres for Horticultural Perishables. Davis. In: Postharvest Technology Research and Information Center, 2001, pp 153.

Kuroki, S., S. Oshita, I. Sotome, Y. Kawagoe and Y. Seo (2004). Postharvest Biology and Technology 33(3), 243-255.

Lammertyn J., N. Scheerlinck, P. Jancsók, B.E. Verlinden, B.M. Nicolaï (2003a). A respiration-diffusion model for 'Conference' pears I: model development and validation. Postharvest Biology and Technology. 30, 31-44.

Lammertyn J., N. Scheerlinck, P. Jancsók, B.E. Verlinden, B.M. Nicolaï (2003b). A respiration-diffusion model for 'Conference' pears: II. Simulations and relation to core breakdown. Postharvest Biology and Technology. 30, 45-57.

Mannapperuma J. D., R.P. Singh, M.E. Montero (1991). Simultaneous gas diffusion and chemical reaction in foods stored in modified atmospheres. Journal of Food Engineering. 14, 167-183.

Nguyen T.A., T. Dresselaers, P. Verboven, G. D'hallewin, N. Culledu, P. Van Hecke, and B.M. Nicolaï (2005). Finite element modelling and MRI validation of 3D transient water profiles in pears during postharvest storage. Journal of the Science of Food and Agriculture. Accepted.

Nicolaï, B.M., P. Verboven, and N. Scheerlinck (2001). The modelling of heat and mass transfer. In: Food Process Modelling (Tijskens L.M.M., M.L.A.T.M. Hertog, B.M. Nicolaï (Eds.)). Woodhead Publishing Limited and CRC Press LLC, Cambridge, UK and Boca Raton, FL, USA, pp. 60-86.

Nobel, P.S. (1991). Physicochemical and Environmental Plant Physiology. Academic Press Inc. San Diego.

Panasenko G. (2005) Multi-scale Modelling for Structures and Composites. Springer.

Schotsmans, W., B.E. Verlinden, J. Lammertyn, B.M. Nicolaï (2003). The relationship between gas transport properties in apple and the histological properties of the apple tissue. Journal of the Science of Food and Agriculture. 84, 1131-1140.

Veltman, R.H., M.G. Sanders, S.T. Persijn, H.W. Peppelenbos and J. Oosterhaven (1999). Decreased ascorbic acid levels and brown core development in pears (Pyrus communis cv. communis). Physiologia Plantarum. 107(1):39-45. 
Veraverbeke, E.A., P. Verboven, P. Van Oostveldt, B.M. Nicolaï (2003a). Prediction of moisture loss across the cuticle of apple (Malus sylvestris subsp. mitis (Wallr.)) during storage Part 1. Model development and determination of diffusion coefficients. Postharvest Biology and Technology. 30, 75-88.

Veraverbeke, E.A., P. Verboven, P. Van Oostveldt, B.M. Nicolaï (2003b). Prediction of moisture loss across the cuticle of apple (Malus sylvestris subsp. mitis (Wallr.)) during storage Part 2. Model validation and sensitivity analysis. Postharvest Biology and Technology. 30, 89-97.

Yearsley C.W., N.H. Banks, S. Ganesh, D.J. Cleland (1996) Determination of lower oxygen limits for apple fruit. Postharvest Biology and Technology. 8, 95-109

Zdunek A., M. Umeda, K. Konstankiewicz (2004). Method of parenchyma cells parametrisation using fluorescence images obtained by confocal scanning laser microscope. Electronic Journal of Polish Agricultural Universities. Agricultural Engineering, Vol. 7(1) 